\title{
Editorial - Von Fall zu Fall: Einblicke in die Praxis
}

\author{
Karin Pinter
}

Online publiziert: 11. November 2015

(C) Springer-Verlag Wien 2015

Der vorliegende Band des psychotherapie forums ist ein offenes Heft mit freien Einreichungen der AutorInnen. Thematisch lässt sich ein roter Faden in den ersten drei Beiträgen finden - und zwar sind dies aufschlussreiche Fallvignetten und Falldarstellungen, eingebettet in theoretische Konzepte, methodische Anwendungen und begleitende reflexive Prozesse. Besondere Bedeutung kommt dem Umstand zu, dass in diesen Fallgeschichten Diskontinuitäten auftreten, die teils Wendepunkte, auf jeden Fall aber komplexe Herausforderungen für die AutorInnen und Chancen für die therapeutische Veränderung darstellen.

Gertraud Diem-Wille beschäftigt sich zunächst ausführlich mit einigen theoretischen Konzepten von Bion, wie etwa das Container-Contained Modell. Sie beleuchtet insbesondere die Relevanz dieser Theorien für den klinischen Kontext. In einer Fallvignette aus der psychoanalytischen Arbeit mit einem dreijährigen Kind zeigt sie auf, wie wir als TherapeutInnen Teil der inneren emotionalen Welt der KlientInnen werden und wie wir diesen Prozess zur Veränderung nutzen können.

Einen Fallbericht über die therapeutische Arbeit mit einem neunjährigen Kind unter Verwendung der Methode der Katathym Imaginativen Psychotherapie bringt Susanne Stefan ein. Sie dokumentiert die erfolgreiche therapeutische Arbeit an Hand von Zeichnungen der Klientin, die den Veränderungsprozess ablesbar und damit die Bedeutung der metaphorischen Welt deutlich machen.

An einer weiteren Falldarstellung lässt uns P. Christian Endler teilhaben. Er erlaubt uns, Einblick in die psychoanalytisch orientierte Fokaltherapie mit einem älteren
Klienten mit einer Demenz-Diagnose zu gewinnen. Mittels ausführlich dokumentierter Stundenprotokolle und parallel dazu geführten Selbstreflexionen versucht der Autor Antworten auf Fragen des Umgangs mit angekündigtem Suizid, der Demenz-Diagnose sowie der Übertragung und Gegenübertragung zu finden.

Zur aktuellen Frage der Abgrenzung von Psychotherapie und alternativen Heilmethoden hat der ÖAGG (Österreichischer Arbeitskreis für Gruppentherapie und Gruppendynamik) eine Befragung unter Auszubildenden im Rahmen des Propädeutikums durchgeführt. Sabine Völkl-Kernstock berichtet über die Ergebnisse dieser österreichischen Studie. Im Wesentlichen zeigt sich eine große Unsicherheit und Ambivalenz der Befragten im Hinblick auf diese Abgrenzung. Die Autorin empfiehlt daher, verstärkt Aufklärung über die Notwendigkeit dieser Abgrenzung in den fachlichen Ausbildungsprozess (Fachspezifika) einzuführen.
K. Pinter $(\square)$

Röntgengasse 7,

1170 Wien, Österreich

E-Mail: karin.pinter@wagnerpinter.at 